\title{
Comparing Survival Curves Using Rank Tests
}

\author{
W. Albers
}

Department of Applied Mathematics University of Twente

\section{Summary}

Survival times of patients can be compared using rank tests in various experimental setups, including the two-sample cuse and the case of paired data. Attention is focussed on two frequently occurring complications in medical applications: censoring and tail alternatives. A review is given of the author's recent work on a new and simple class of censored rank tests. Various models for tail alternatives are discussed and the relation to censoring is demonstrated.

Key words: Censoring; Tail alternatives; Efficiency.

\section{Introduction}

Consider the standard question whether there exists a systematic difference between the survival times for two groups of patients which have received different treatments. Hence we have a sample $X_{1}, \ldots, X_{m}$ from a continuous distribution function (df) $F(x)$ and, independently, a sample $X_{m+1}, \ldots, X_{m+n}$ from a continuous df $F(x)$. Typically, it is supposed that the difference between the two treatments is constant in the sense that $F(x)=F(x-\theta)$ for some parameter $\theta$. The question is then settled by testing the hypothesis $H_{0}: \theta=0$ against e.g. the alternative $H_{1}: \theta>0$. If in addition it is assumed that $F$ is normal, we have arrived at one of the most common statistical problems, for which Student's two-sample $t$-test is the well-known solution.

However, quite often the normality assumption is not warranted and we prefer a distributionfree test, typically a rank test. Rearrange all $N=m+n$ observations into increasing order of magnitude and record the ranks $R_{1}, \ldots, R_{N}$ of the original $X_{1}, \ldots, X_{N}$ in this sequence. The most familiar two-sample rank test is Wilcoxon's test, which rejects $H_{0}$ in favor of $H_{1}$ for large values of

$$
\sum_{j=m+1}^{N} R_{j}
$$

Wilcoxon's test is but one example from a whole family of rank tests. In fact, let $J$ be a so-called score function on the unit interval, then we have the general $11^{*}$ 
two-sample rank statistic

$$
T=\sum_{j=m+1}^{N} J\left(\frac{R_{j}}{N+1}\right)
$$

Obviously, $J(t)=t$ reproduces the Wilcoxon case. The rationale behind the generalization in (1.2) is the following: it can be shown (see e.g. HÁJEK and Š́ID́K (1967)) that to each type of underlying $\mathrm{d} f F$ there corresponds precisely one choice, which we will denote by $\psi$, for the score function $J$ that is optimal in the sense that the rank test based on this $\psi$ is most powerful against location alternatives $F(x-\theta)$. The Wilcoxon-type scores from $J(t)=t$ e.g. are optimal against logistic alternatives $(F(x)=1 /\{1+\exp (-x)\})$.

To keep our exposition as simple and explicit as possible, we shall put little emphasis on the general case and instead concentrate on a few particular choices that are widely used in practice. To be specific, in addition to the Wilcoxon-type scores we shall encounter scores based on $J(t)=-\log (1-t)$. The corresponding $\log$-rank test is optimal for $F(x)=1-\exp \left(-e^{x}\right)$. This hardly explains its apparent attractiveness in practice and therefore some additional explanation is required. Besides the most frequently used location or shift alternatives $\hat{F}(x)=F(x-\theta)$ considered up to now, it can also be of interest to consider scale alternatives $F(x)=F(x /(1+\theta))$. This is especially the case for non-negative observations, such as the survival times under consideration here. In this connection the exponential d $F(x)=1-e^{-\lambda x}(x>0)$ is of special interest as its so-called hazard rate $f(x) /(1-F(x))$ is constant. The choice $J(t)=-\log (1-t)$ now turns out to be optimal for scale alternatives from this exponential distribution (see once more HÁJEK and SIDAK, 1967). The corresponding test incidentally also is known as the Savage test, based on the scores $\sum_{j=N+1-k}^{N}(1 / j)$, which are asymptotically equivalent to the scores $-\log (1-k /(N+1))$ obtained from $J(t)$.

A final remark is that it often proves convenient to standardize the scores such that their total sum equals 0 , which obviously requires $\int_{0}^{1} J(t) \mathrm{d} t=0$. Hence in the sequel we shall apply Wilcoxon-type and Savage-type scores derived from

$$
J_{w}(t)=2 t-1, \quad J_{s}(t)=-1-\log (1-t),
$$

respectively.

Having introduced the standard situation, we now turn to the complications of interest in this paper. Especially in medical applications, it frequently occurs that the data become only partly available: due to competing risks (death due to other causes, moving to another part of the country, etc.), patients are withdrawn from the trial. Hence their survival times are censored: they are merely known to exceed a certain number, the censoring time. In recent years a lot of effort has been devoted to adapting rank tests to such censored data situations. Typically, the resulting procedures involve rather sophisticated mathematics 
in their derivation and, more seriously, show little resemblance to the original rank statistic from (1.2). Here we shall discuss a new class of censored data rank tests, based on the idea of treating the uncensored and the censored observations as two separate groups for each of which an appropriate statistic of the form (1.2) is obtained. A suitable combination of these two rank statistics then produces the final test statistic. Advantages of this approach are: (i) it is relatively easy to interpret and to apply, (ii) the test is closely related to uncensored rank tests and (iii) its behaviour is simply analyzed using standard rank test theory.

In section 2 we shall cover the two-sample problem, while in section 3 we shall discuss the case of paired data. Readers interested in the technical background are referred to Albers and Akritas (1087) for as far as section 2 is concerned, and to Albers (1988a) for section 3. In these papers, the general problem is attacked, rather than some widely used examples. More importantly, the emphasis there is on the methodological aspects, such as the connection to uncensored rank tests and the derivation of asymptotic properties. The present exposition focusses on demonstrating the relevance of such results for medical applications.

The second complication considered concerns tail alternatives. In the above we already observed that location alternatives $F(x)=F(x-\theta)$ are not always satisfactory and that sometimes we prefer scale alternatives $\widehat{F}(x)=F(x /(1+\theta))$. But clearly this is not the end of it: many other interesting classes of alternatives can be thought of. One such class consists of so-called tail alternatives, which arise when substantial differences exist between survival distributions later in time but fail to exist earlier in time. This phenomenon is quite common in medical applications, see e.g. Flemivg et al. (1980) p. 608, who mention a.o. "certain treatments for coronary heart disease yielding remarkably improved long-term survival, even though survival immediately following onset of treatment may be worse than that obtained with less aggressive treatments." In section 4 we shall discuss some approaches for modeling such tail alternatives and reveal the similarities which exist between adapting rank tests to censoring on one hand, and to tail alternatives on the other. This is especially helpful for analyzing situations where both complications arise at the same time.

\section{Two-Sample Censored Rank Tests}

Before we introduce the appropriate censorship model we briefly mention a considerably simpler model to help understand what happens in the general case. This simple case concerns Type II censoring. Here only the smallest $k$ of the $N=m+n$ ordered observations are available. This situation one typically encounters frequently in industrial experiments: rather than using sample size $k$ and waiting till the last item has expired, one uses $N>k$ items and just waits until the $k$-th item has expired. The optimal strategy in this situation is still easy to guess. For the uncensored observations continue to use the aforemen- 
tioned optimal score function $J=\psi$, while the censored (i.e. the final $N-k$ ) observations will necessarily have to get the same score, as their order remains unknown. A fair choice for this constant score obviously is the average of the scores still available at the time of censoring. Note that it boils down to replacing $\psi$ on $(p, 1)$, where $p=k / N$, by its right-hand average

$$
\chi(p)=\int_{j}^{1} \psi(u) \mathrm{d} u /(1-p),
$$

(see e.g. Gastwirth, 1965). The resemblance to uncensored rank tests is still evident and the standard theory continues to be applicable.

Unfortunately, this breaks down if we move on to more complicated, and for our purposes more realistic, censoring mechanisms like progressive Type II censoring or random censoring. Then the tests introduced by Prentice (1978) are optimal. They still use scores derived from $\psi$ for the uncensored observations and scores derived from the right-hand average $\chi$ in (2.1) for the censored ones. But matters are complicated by the occurrence of the so-called Kaplan-Meier estimate for the underlying $\mathrm{d} f F$ in the scores. Moreover, standard rank test theory is no longer applicable.

To overcome these problems, Albers and Akritas (1987) (to be denoted by $A A$ in the sequel) have introduced a new class of tests to be applied under the random censorship model. Next to and independent of the two samples $X_{1}, \ldots$, $X_{m}, X_{m+1}, \ldots, X_{N}$ of survival times, we have independent censoring times $W_{1}, \ldots$, $W_{N}$ from a single $\mathrm{d} f D$. This assumption of equal censorship for both samples is vital for our approach. Now the trial will not produce the actual survival times $X_{j}$, but merely the pairs $\left(\mathbb{X}_{j}, V_{j}\right)$, where

$$
\left\{\begin{array}{l}
\bar{X}_{j}=\min \left(X_{j}, W_{j}\right) \\
V_{j}=1 \quad \text { if } \quad \bar{X}_{j}=X_{j} \text { and } \quad V_{j}=0 \text { else. }
\end{array}\right.
$$

So for each patient we get a time, together with the information whether this is his or her survival time, or the time at which censoring has occurred. Hence we have a kind of $2 \times 2$ table: there are two groups of patients, each of which is divided into censored and uncensored observations. The idea now is to apply two separate two-sample rank statistics, one for comparing the two groups of uncensored observations, the other for comparing the two groups of censored observations. At first sight this splitting of the problem might seem to lead to an unacceptable loss of power, but in fact it can be shown that this is not the case: the loss is asymptotically negligible if we simply add the two separate statistics! (see AA for technical details.)

Hence, let

$$
K=\sum_{j=1}^{m} V_{j}, \quad L=\sum_{j=m+1}^{N} V_{j}
$$

be the number of uncensored observations from the first and second sample, respectively. Given $(K, L)=(k, l)$, let $R_{1}, \ldots, R_{\mathrm{k}+l}$ and $Q_{1}, \ldots, Q_{N-\mathrm{k}-l}$ be the ranks 
of the $(k+l)$ uncensored observations and the $(N-k-l)$ censored observations, respectively. Then in analogy to (1.2) we have the combined rank statistic

$$
T^{*}=\sum_{j=K+1}^{K+L} J_{1}\left(\frac{R_{j}}{K+L+1}\right)+\sum_{j=m-K+1}^{N-K-L} J_{0}\left(\frac{Q_{j}}{N-K-L+1}\right)
$$

where $J_{1}$ and $J_{0}$ are appropriate score functions.

The first step towards optimality is achieved once more by letting $J_{1}=\psi$ and $J_{0}=\chi$ as in (2.1). However, we should realize that even the uncensored observations no longer are ordinary observations from the df $F$ : we are dealing with $X_{j}$, given that $\min \left(X_{j}, W_{j}\right)=X_{j}$, and hence their distribution is affected by the censoring distribution $D$. Hence the final result for the optimal choices is of the form

$$
J_{1}(t)=\psi(\Gamma(t)), \quad J_{0}(t)=\chi(\Lambda(t)),
$$

where $\Gamma$ and $A$ are determined by the way $D$ depends on $F$ (see again $A A$ for details).

Here we concentrate on the two examples from (1.3). The Wilcoxon choice $\varphi(t)=J_{w}(t)=(2 t-1)$ leads through $(2.1)$ to $\chi(t)=t$. Note that this is easy to interpret: death very early in time results in a negative weight close to -1 , very late in time to almost +1 , while in the middle we have 0 . Likewise, censoring near the onset provides almost no information and the weight should be near 0 , as is the case with $\chi(t)=t$. On the other hand, censoring very late in time destroys little information and a weight near 1 is appropriate, as is indeed the case since $t$ and $2 t-1$ become close. For the choice $\psi(t)=J_{s}(t)=-1-\log (1-t)$ we obtain $\chi(t)=-\log (1-t)$ and similar remarks apply.

Let $\bar{H}$ denote $1-H$ for any df $H$, then a familiar way of modeling the dependence between $D$ and $F$ is to let $\bar{D}$ be a power of $\vec{F}$. Then it can be shown that

$$
\Gamma(t)=\Lambda(t)=1-(1-t)^{x},
$$

where the parameter $\tau$ is precisely the probability of an observation being uncensored. (Hence for $\tau=1$ we get $\Gamma(t)=\Lambda(t)=t$, as should be the case.) Combination with the above leads to

$$
\begin{aligned}
J_{W 1}(t) & =1-2(1-t)^{\tau}, & J_{1{ }^{0}}(t) & =1-(1-t)^{\tau}, \\
J_{S 1}(t) & =-1-\tau \log (1-t), & J_{S 0}(t) & =-\tau \log (1-t) .
\end{aligned}
$$

The asymptotic properties of the test based on (2.4) follow easily from standard rank test theory. As an example, the Wilcoxon-type scores under (2.6) and logistic alternatives lead to a $T^{*}$ which is asymptotically normal with mean $\theta \mu$ and variance $\mu$, where $\mu=m n \tau /(N(1+2 \tau))$. The parameter $\tau$ is typically unknown of course, but it can be estimated efficiently by

$$
S=\frac{K+L}{N},
$$

with $K$ and $L$ as in (2.3). It can be shown that under (2.5) the test is asymptoti- 
cally equivalent to Prentice's test, which is asymptotically optimal. Both simulations and exact efficiency computations show that the test works quite well. In the situation considered in the next section we shall present a real data example.

\section{Rank Tests for Censored Paired Data}

Up to now we have restricted attention to the two-sample problem, but this is by no means the only design where censored rank tests are applicable. In this section we consider the case where the two groups are not independent, but rather consist of matched pairs of patients. In the uncensored case one typically proceeds as follows: on the basis of the differences between the observations within each pair a one-sample test is performed to test whether the $\mathrm{d} f$ of these differences has mean zero. Under normality, the one-sample $t$-test is used. Otherwise, one could use e.g. Wilcoxon's signed rank test.

It does not require a lot of thought to realize that this approach is difficult to generalize to the censored case. Fortunately, an alternative starting point is available: rather than using tests based on ranks of differences, one can use tests based on differences of ranks. In fact, one essentially uses the two-sample test of section 2, but adapts its variance to take the dependence into account. (See a.o. SniJders (1981).)

To introduce the test, we first adapt the notation to the present design: let $\left(X_{11}, X_{21}\right), \ldots,\left(X_{1 n}, X_{2 n}\right)$ be $n$ independent pairs of survival times from a continuous bivariate $\mathrm{d} f$. Suppose $X_{11}$ has marginal $\mathrm{d} f F(x)$ and $X_{21}$ has marginal d $f F(x-\theta)$. Then again we want to test $H_{0}: \theta=0$ against $H_{1}: \theta>0$. Denote the ranks of $X_{11}, \ldots, X_{1 n}, X_{21}, \ldots, X_{2 n}$ in the pooled sample by $R_{11}, \ldots, R_{1 n}, R_{21}, \ldots, R_{2 n}$, then in analogy to (1.2) we arrive at the rank statistic for the uncensored case $T=\sum_{j=1}^{n} J\left(R_{2 j} /(2 n+1)\right)$. However, it is more illuninating to work with the equivalent statistic $T=2 T-\sum_{i=1}^{2 n} J(i /(2 n+1))$, which can be written as

$$
T=\sum_{j=1}^{n}\left\{J\left(\frac{R_{2 j}}{2 n+1}\right)-J\left(\frac{R_{1 j}}{2 n+1}\right)\right\},
$$

and as such clearly locates the effect of the dependence within the pairs in the individual summands of $T$. In this way it is also easily verified that the variance of $T$ has to be corrected compared to the independent case by a factor $\left\{1-\varrho\left(J\left(F\left(X_{21}\right)\right), J\left(F\left(X_{11}\right)\right)\right)\right\}$.

In complete analogy to section 2 we now add the complication of random censorship to the picture. For the resulting situation, ALBERs (1988a) has derived a censored paired rank test from (3.1). Let $V_{i j}, i=1,2, j=1, \ldots, n$ again be the indicators of censoring (cf. (2.2)) and let $L=\sum_{j=1}^{n}\left(V_{1 j}+V_{2 j}\right)$. As in the two-sample case, rank the $L$ uncensored and the $(2 n-L)$ censored observations separately. 
Denote the rank of $X_{i j}$ by $R_{i j}\left(V_{i j}\right)$ and its standardized version by $R_{i j}^{*}\left(\nabla_{i j}\right)$. (Hence $R_{i j}^{*}(1)=R_{i j}(1) /(L+1)$ and $R_{i j}^{*}(0)=R_{i j}(0) /(2 n-L+1)$.) Then, in analogy to the step from (1.2) to (2.4), we go from (3.1) to

$$
T^{*}=\sum_{j=1}^{n}\left\{J_{\nabla_{2 j}}\left(R_{2 j}^{*}\left(\nabla_{2 j}\right)\right)-J_{V_{1 j}}\left(R_{1 j}^{*}\left(V_{1 j}\right)\right)\right\}
$$

By way of illustration we quote an example from ALBERS (1988a) based on data from O'Brien and Fleming (1987), concerning the survival times for skin grafts on 11 patients, each of whom received both a closely and a poorly matched graft. In Table 1 it is shown how the 20 uncensored and the 2 censored observa-

\section{Table 1}

Observed Survival Times to Skin Grafts on Burn Patients

\begin{tabular}{llllccc}
\hline $\begin{array}{l}\text { Patient } \\
\text { number }\end{array}$ & $\begin{array}{l}\text { Close } \\
\text { match } x_{2 j}\end{array}$ & $\begin{array}{l}\text { Poor } \\
\text { match } x_{1 j}\end{array}$ & $\begin{array}{l}\text { Rinks } \\
r_{2 j}(1)\end{array}$ & $r_{2 j}(0)$ & $r_{1 j}(1)$ & $r_{1 j}(0)$ \\
\hline 1 & 37 & 29 & 16 & & 4.5 \\
2 & 19 & 13 & 8 & & 2 \\
3 & $57^{*}$ & 15 & & 1 & 3.5 \\
4 & 93 & 26 & 20 & & 12.5 \\
5 & 16 & 11 & 5 & & 1 & \\
6 & 22 & 17 & 11 & & 12.5 \\
7 & 20 & 26 & 9 & & 10 \\
8 & 18 & 21 & 7 & & 18 \\
9 & 63 & 43 & 19 & & 3.5 \\
10 & 29 & 15 & 14.5 & & 17
\end{tabular}

- Indicates the survival time was censored.

tions are ranked separately. According to (2.8), we can estimate $\tau$ by $s=20 / 22$ here. Using the choices in (2.7) we obtain from (3.2) for the Wilcoxon- and Savagetype statistics the values 3.830 and 5.531 , respectively. The null variance of the statistics can be estimated by $\sum_{j=1}^{n}\left\{J_{V_{2 j}}\left(R_{2 j}^{*}\left(V_{2 j}\right)\right)-J_{V_{1 j}}\left(R_{1 j}^{*}\left(V_{1 j}\right)\right)\right\}^{2}$, leading to 3.415 and 6.919, respectively, resulting in standardized values 2.073 and 2.103 and $P$ values .019 and .018 , respectively. By way of comparison, we mention that O'Brien and Fleming (1987) obtain a $P$ value of .017 for the paired data version of the Wilcoxon-type Prentice test they consider.

In this section we have discussed how two-sample results can be adapted to the paired data case. In a similar fashion, extensions can be made to the $k$ sample case and the regression problem. See ALBERS (1988b). 


\section{Tail Alternatives}

As stated in the introduction, tail alternatives occur when differences in survival distributions are substantial later in time, but fail to exist earlier on. A first possibility which springs to mind in looking for a way to model such a phenomenon, is to take an ordinary location or scale alternative as a starting point and to subsequently contract the difference between the two distributions from $(-\infty, \infty)$ (or $(0, \infty)$ in the scale case) onto $\left(x_{0}, \infty\right)$, for some time point $x_{0}$. An example of this nature can be found in Fleming et al. (1980), p. 619: consider the exponential scale alternatives discussed before, which have constant hazard rate. In the ordinary scale case we have (in our notation) rate $\lambda$ under $H_{0}$ and rate $\lambda /(1+\theta)$ under $H_{1}$. To create a tail alternative, we let this situation continue to exist only beyond $x_{0}$, and we replace the rate on $\left(0, x_{0}\right)$ by a common value, e.g. $\lambda$. A similar idea was used by Mason and Schuenemeyer (1983) in introducing "a convenient class of tail alternatives" (p. 936).

It is easy to verify that the contraction mentioned above should be tranşlated into a contraction of the score function: if $\psi(t)$ is optimal for the parent model, then it is optimal to use for the tail alternative a $J$ of the form

$$
J(t)= \begin{cases}0 & t \leqq a, \\ \psi\left(\frac{t-a}{1-a}\right), & t>a,\end{cases}
$$

for some $a$ with $0 \leqq a<1$. For Wilcoxon-type scores this leads to 0 on $(0, a]$ and $1-2(1-t) /(1-a)$ on $(a, 1)$. A similar result holds for Savage type scores.

An obvious disadvantage of this type of score function is its jumpy character. Typically, $\psi$ increases smoothly from some negative value in 0 towards a positive value in 1 . Hence in the parent model the scores also increase smoothly. But in the contraction model one should assign score zero to all patients up to a certain point. Then, the first patient after this moment has bad luck: to him or her the lowest possible score is assigned. Next the scores start to climb again and eventually become positive. One could argue that we need not worry about this peculiar behaviour as long as it is optimal. However, the point is that the optimality depends on the typically unknown starting point $a$. It is intuitively clear and can be verified through computation, that the efficiency deteriorates rapidly if the $a$ chosen in (4.1) becomes different from the true value.

Hence it makes sense to look for alternative ways of modeling tail alternatives. A possible approach is to generalize the shift model $F(x)=F(x-\theta)$ along the lines indicated by e.g. Doksum (1974). Let $X$ and $Y$ be the survival time of a patient from the first and the second group, repectively, then $Y$ is distributed as $X+\theta$ in the shift model. A more general model results if we let $Y$ and $X+\theta G(X)$ be equally distributed, for some shift function $G$. To obtain tail alternatives, we concentrate on nonnegative, nondecreasing $G$ which are small early in time. Then it becomes possible, and will turn out to be convenient, to write $G(x)$ as 
$B(F(x))$ for some nonnegative, nondecreasing function $B$ on $(0,1)$. Hence

$$
Y \text { and } X+\theta B(F(X))
$$

are equally distributed. A choice like $B(t)=t^{r}, r=0,1,2, \ldots$ illustrates the idea: for $r=0$, we are back at the shift model, while for $r$ increasing the tail character becomes more pronounced.

Again let $\psi$ be the optimal score function for the parent model. It can be verified that the introduction of $B$ in (4.2) then leads to an optimal $J$ determined by

$$
\int_{0}^{t} J(u) \mathrm{d} u=B(t) \int_{0}^{t} \psi(u) \mathrm{d} u .
$$

The obvious example is obtained by choosing once more $\psi(t)=2 t-1$ and by letting $B(t)=t^{r}$. This leads to $J(t)=t^{r}\{(r+2)-t(r+1)\}$, which decreases from 0 to $-c^{r}$ at $c=r /(r+2)$, and subsequently increases to 1 , passing 0 at $(r+1) /(r+2)$. Note that the behaviour of this $J$ is somewhat similar to that of the example following (4.1). However, the present choice is much smoother. Additional illustration of this point is obtained by determining the function $B$ in (4.3) which leads precisely to the transformation (4.1). It turns out that for the Wilcoxon case $B(t)=(1-a / t) /(1-a)$ on $(a, 1)$, and 0 otherwise. This shift function is nonsmooth: the survival distributions agree exactly up to a point, after which a rapidly growing discrepancy occurs.

The final issue we address here is the relation to censoring. In both (4.1) and (4.3) we observed that modifying the model towards tail alternatives induced a transformation of the score function $\psi$. But censoring has exactly the same effect! To be more precise, in (2.5) we obtained the score function $J_{1}(t)=\psi(\Gamma(t))$ for the uncensored observations, leading to Wilcoxon-type scores $J_{\mathrm{w} 1}(t)=$ $=1-2(1-t)^{\mathrm{r}}$ in (2.7). As usual (cf. (1.3)) standardize $J_{1}(t)$ to $J_{1}(t)=J_{1}(t)-\int_{0}^{1} J_{1}(u) \mathrm{d} u$, which has $\int_{0}^{1} J_{1}(t) d t=0$. Then it follows from (4.3) that censoring has the same effect as introducing a shift function

$$
B(t)=\int_{0}^{t} J_{1}(u) \mathrm{d} u / \int_{0}^{t} \psi(u) \mathrm{d} u .
$$

In the Wilcoxon case $B(t)$ is proportional to $\{1-(1-t) r\} / t$, which increases smoothly from the value $\tau$ at 0 to the value 1 at 1 , and thus represents a very mild form of tail alternative.

In view of the similarities observed, it is now also clear what to do if both complications occur together: the survival times can be censored and the corresponding distributions differ mainly in the tails. Then we just apply a double transformation to $\psi$ to take each of these effects into acount. An example: using $J_{\mathrm{W} 1}(t)=1-2(1-t)^{\text {ar }}$ for $0<\alpha \leqq 1$ is adequate for censoring at rate $\tau$ and tail alternative with shift function $B(t)=\left\{1-(1-t)^{\alpha}\right\} / t$. 
References

ALBers, W., 1988a: Combined rank tests for randomly censored paired data. J. Amer. Statist. Assoc. 83, 1159-1162.

ALBmBs, W., 1988b: Rank tests for regression and $k$-sample rank tests under random censorship. Statistics and Probability Letters 6, 315-319.

ALBzrs, W., and ARritas, M. G., 1987: Combined rank tests for the two-sample problem with randomly censored data. J. Amer. Statist. Assoc. 82, 648-655.

Dorsum, K., 1974: Empirical probability plots and statistical inference for nonlinear models in the two-sample case. Ann. Statist. 2, 267-277.

Flemmia, T. R., O'Falion, J. R., O. Brien, P. C. and Harrington, D. P., 1980: Modified Kolmogorov-Smirnov test procedures with application to arbitrarily right-censored data. Biometrics 36, 607-625.

GASTwIRTH, J. L., 1965: Asymptotically most powerful rank tests for the two-sample problem with censored data. Ann. Math. Statist. 36, 1243-1247.

HÁJEK, J., and ŠroňK, Z., 1967: Theory of Rank Tests. New York: Academic Press.

Mason, D. M., and Schugnemryer, J. H., 1983: A modified Kolmogorov-Smirnov test sensitive to tail alternatives. Ann. Statist. 11, 933-946.

O'Brign, P. C., and Flemona, T. R., 1987: A paired Prentice-Wilcoxon test for censored paired datr. Biometrics 43, 169-180.

PRentice, R. L., 1978: Linear rank tests with right censored data. Biometrika 65, 167-179.

SNIJDERS, T., 1981 : Rank tests for bivariate symmetry. Ann. Statist. 9, 1087-1095.

Received Jan. 1990

Revised March 1990

W. Albers

Department of Applied Mathematics

University of Twente

P.O. Box 217

7500 AE Enschede, The Netherlands 